\title{
Análisis de la motivación en las clases de Educación Física en Primaria
}

Emilio Dorado Cuevas', Gema Paramio-Pérez² y Bartolomé J. Almagro²

1 Graduado en Educación Primaria, con Mención en EF (Universidad de Huelva)

2 Universidad de Huelva

Email: almagro@dempc.uhu.es

\section{RESUMEN:}

El objetivo principal de este trabajo fue analizar la motivación del alumnado de Educación Física en Primaria. Se utilizó una muestra conformada por 94 alumnos de un centro escolar público de Huelva, de los cuales 47 son niños y 47 niñas, con una media de edad de 10.87 años. Se empleó un cuestionario para medir los diferentes tipos de motivación que establece la teoría de la autodeterminación, así como otros datos socio-demográficos y si realizaban actividades físicodeportivas extraescolares. Los resultados de este estudio han mostrado que la motivación en las clases de EF del alumnado encuestado es positiva, es decir, obtuvieron mayores puntuaciones en las formas de motivación más autodeterminadas. Además, se encontraron diferencias estadísticamente significativas en la motivación intrínseca a favor de las alumnas. En cuanto al alumnado que practicaba alguna actividad deportiva fuera del centro, los deportes más practicados fueron: el fútbol, el baloncesto y la natación. Por último, el $25.5 \%$ de alumnado no realizaba ninguna actividad física o deportiva extraescolar.

PALABRAS CLAVE: Teoría de la Autodeterminación, Actividades Extraescolares, Alumnado, Estrategias Motivacionales. 


\section{INTRODUCCIÓN}

La motivación en la Educación Física escolar puede considerarse un tema de estudio muy interesante, puesto que en la actualidad hay muchos jóvenes que la única actividad físico-deportiva que practican, la realizan durante las clases de Educación Física (en adelante EF). En este sentido, la evidencia científica ha mostrado que la motivación experimentada en las clases de EF es un aspecto clave para que los alumnos mantengan su intención de seguir siendo físicamente activo (Almagro, Navarro, Paramio, y Saénz-López, 2015; Méndez-Giménez, Cecchini, Fernández-Río, y González, 2012).

Para ayudarnos a entender mejor los procesos motivacionales que tiene lugar en las clases de EF, los investigadores han empleado fundamentalmente la teoría de la autodeterminación (Deci y Ryan, 1985, 2000, 2012, 2014; Ryan y Deci, 2000). La teoría de la autodeterminación es una macro-teoría de la motivación humana (Deci y Ryan, 2008) que, hasta la actualidad, está formada por seis mini-teorías (Deci y Ryan, 2014). En concreto, durante las últimas tres décadas, se han desarrollado seis miniteorías para abordar diferentes cuestiones: los efectos de los factores sociales en la motivación intrínseca (teoría de la evaluación cognitiva; Deci y Ryan, 1985, 2000); el desarrollo de la motivación extrínseca y los factores contextuales que promueven o impiden la interiorización e integración de éstos en la regulación de las conductas (teoría de la integración orgánica; Deci y Ryan, 1985, 2000); las diferencias individuales en las orientaciones motivacionales (teoría de la orientaciones de causalidad; Deci y Ryan, 1985, 2000); el funcionamiento de las necesidades psicológicas básicas que son esenciales para el crecimiento, la integridad y el bienestar (teoría de las necesidades básicas; Deci y Ryan, 1985, 2000); los efectos de diferentes contenidos de meta en el bienestar y el rendimiento (teoría de los contenidos de meta; Ryan, Williams, Patrick, y Deci, 2009; Vansteenkiste, Niemiec, y Soenens, 2010); y la importancia del desarrollo y mantenimiento de las relaciones personales cercanas para favorecer la calidad de las mismas, el ajuste y el bienestar (teoría motivacional de las relaciones; Deci y Ryan, 2014).

Esta investigación se fundamenta principalmente en la teoría de la integración orgánica. Esta mini-teoría de la teoría de la autodeterminación (TAD) establece diferentes tipos de motivación a lo largo de un continuo, en función del nivel de autodeterminación de la conducta (Deci y Ryan, 2000; Ryan y Deci, 2000). Según la TAD (Ryan y Deci, 2000) se pueden encontrar, de menor a mayor autodeterminación, tres tipos de motivación: la desmotivación, la motivación extrínseca (regulación externa, regulación introyectada, regulación identificada y regulación integrada) y la motivación intrínseca. En la desmotivación, a los alumnos les falta intención de actuar (Deci y Ryan, 2000), y por tanto, es probable que su participación en clases sea desorganizada y acompañada de sentimientos de frustración, apatía, etc. La motivación extrínseca está determinada por recompensas o agentes externos, y según esta teoría puede variar bastante en su autonomía relativa. En este sentido, un alumno podría participar en las clases de EF por presiones externas (regulación externa), por sentimientos de culpabilidad (regulación introyectada), porque quiere mejorar sus habilidades deportivas (regulación identificada) o porque sabe que es necesario para mantener un estilo de vida saludable (regulación integrada). Sin embargo, la regulación integrada no parece estar presente en jóvenes (Vallerand y Rousseau, 2001). En este sentido, en esta investigación se empleó una escala que no mide este último tipo de regulación, ya que la edad del alumnado estuvo comprendida entre los 10 y los 12 años. Por último, un alumno motivado intrínsecamente participa en las clases de EF por la satisfacción y el placer inherente de la propia actividad. 
Empleando los diferentes tipos de motivación establecidos por la TAD (Deci y Ryan, 2000; Ryan y Deci, 2000), se han realizado diferentes estudios que han relacionado la motivación experimentada en clases de EF con múltiples consecuencias: con la diversión (Moreno, Hernández, y González-Cutre, 2009) y el aburrimiento (Méndez-Giménez, Fernández-Río, y Cecchini-Estrada, 2013), con la importancia concedida a la EF (Moreno, González-Cutre, Ruiz, 2009), con la intención de seguir practicando actividades físico-deportivas fuera del centro (Hein, Müür, y Koka, 2004; Lim y Wang, 2009), con el rendimiento académico y la autoestima (Almagro et al., 2015), etcétera. Esto no hace más que evidenciar la importancia de tratar de evaluar y mejorar la motivación del alumnado en sus clases de EF.

La motivación en el área de Educación Física, es un tema que ha suscitado mucho interés, como podemos apreciar en los numerosos estudios que podemos encontrar en la literatura científica (González-Cutre, Sicilia, y Moreno, 2011; Gutiérrez y López, 2012; Ntoumanis y Standage, 2009; Moreno et al., 2008). Sin embargo, todavía son escasos los estudios centrados en la motivación del alumnado de Educación Física de Primaria (e.g., Cecchini, Fernández-Losa, González, Fernández, y Méndez, 2012; Cecchini, Fernández-Losa, González, y Cecchini, 2013). Por ello, el objetivo principal de este estudio fue analizar la motivación del alumnado de Educación Física en Primaria.

\section{MÉTODO}

\subsection{Participantes}

El estudio se llevó a cabo con una muestra total 94 alumnos y alumnas de un Centro Público de Educación Infantil y Primaria de la ciudad de Huelva, de los cuales 47 eran niños y 47 eran niñas, con edades comprendidas entre 10 y 12 años, siendo la edad media de los mismos de 10.48 años $(D T=.75)$. El muestreo se realizó de forma intencional para seleccionar a discentes con una capacidad cognitiva y madurativa idónea para poder realizar el estudio (por lo que se administró el cuestionario al alumnado de 5ํㅡ y 6을

La participación en el estudio, por parte del alumnado, fue voluntaria y anónima. Además, al ser menores de edad, sus padres los autorizaron a participar. Dichos alumnados rellenaron el cuestionario en un periodo de 15 minutos en la clase de Educación Física, donde el docente de EF estuvo presente junto al autor principal de este trabajo, por si surgía alguna duda sobre los mismos.

\subsection{Instrumentos}

El alumnado que participó en el estudio rellenó un cuestionario que incluía preguntas sobre datos socio-demográficos (genero, edad, curso, práctica de deporte 0 actividad física extraescolar, etc.) y una escala para medir la motivación del alumnado en clases de EF que se describe a continuación.

Escala del Locus Percibido de Causalidad en Educación Física (PLOC). Se empleó la versión testada en el contexto español de Moreno, González- Cutre y Chillón (2009) del cuestionario Perceived Locus of Causality (Goudas, Biddle, y Fox, 1994). Este cuestionario está formado por un total de 20 ítems agrupados y divididos en 5 dimensiones, que son la motivación intrínseca (4 ítems), la regulación identificada 
(4 ítems), la regulación introyectada (4 ítems), la regulación externa (4 ítems) y la desmotivación (4 ítems). La sentencia previa fue "Participo en esta clase de Educación Física...". Las respuestas estaban puntuadas en una escala tipo Likert, con un rango de puntuación que oscilaba entre 1 (Totalmente en desacuerdo) y 7 (Totalmente de acuerdo). Los alfas de Cronbach obtenidos fueron de .67 para la motivación intrínseca, .76 para la regulación identificada, .71 para la regulación introyectada, .71 para la regulación externa y .74 para la desmotivación.

\subsection{Procedimiento}

Para poder llevar a cabo la recogida de datos, se contactó con el equipo directivo para solicitar la autorización del centro. Además, se solicitó la colaboración al especialista de Educación Física del centro (ya que los cuestionarios fueron administrados durante sus clases).

Por otro lado, se les requirió a los padres la autorización escrita para que sus hijos/as participaran en el estudio, informando de la función de dicho cuestionario. Asimismo, se les comunicó que la administración del cuestionario tendría una duración de unos 15 minutos aproximadamente y se garantizó el anonimato de los participantes en dicho estudio.

Por último, la administración de los cuestionarios se llevó a cabo con la presencia del autor principal de este artículo, para intentar resolver cualquier tipo de duda que pudiera tener el alumnado y haciéndoles ver la importancia que tenía el rellenar los cuestionarios de manera sincera, ya que podían ayudar a mejorar las clases de EF gracias a sus respuestas.

\subsection{Análisis de datos}

En primer lugar, se depuró la matriz de datos y se realizó el análisis de la fiabilidad. Posteriormente, se calcularon los estadísticos descriptivos de las diferentes variables de estudio y las correlaciones bivariadas. Por último, se realizó un análisis para comparar las diferencias que hay en la motivación en referencia al sexo de los sujetos (prueba t para muestras independientes). Los diferentes análisis se llevaron a cabo con los paquetes estadísticos SPSS 22.0.

\section{RESULTADOS}

\subsection{Estadísticos descriptivos y correlaciones bivariadas de los diferentes tipos de motivación}

Se calcularon los estadísticos descriptivos de las diferentes regulaciones motivacionales que permite medir la escala empleada (PLOC). Las puntuaciones medias más altas las obtuvieron la motivación intrínseca $(M=6.13)$ y la regulación identificada $(M=6.34)$. Por tanto, se puede afirmar que se obtuvo mayor media en las formas de motivación más autoderterminadas (ver Tabla 1).

El análisis de correlación mostró que la motivación intrínseca correlacionó de forma positiva y significativa con la regulación identificada (el tipo de motivación extrínseca más autodeterminada) y con la regulación introyectada. Además, la 
regulación introyectada y la regulación externa correlacionaron positiva y significativamente con la desmotivación.

Tabla 1. Estadísticas descriptivas y correlaciones entre los diferentes tipos de motivación

\begin{tabular}{lccccccc}
\hline \multicolumn{1}{c}{ Variables } & $\boldsymbol{M}$ & $\mathbf{D T}$ & $\mathbf{1}$ & $\mathbf{2}$ & $\mathbf{3}$ & $\mathbf{4}$ & $\mathbf{5}$ \\
\hline 1. M. Intrínseca & 6.13 & .92 & - & $.54^{* *}$ & $.28^{\star *}$ & .10 & -.13 \\
2. R. Identificada & 6.34 & .64 & & - & $.32^{\star *}$ & .11 & -.09 \\
3. R. Introyectada & 4.11 & 1.50 & & & - & $.46^{\star *}$ & $.24^{*}$ \\
4. R. Externa & 3.35 & 1.50 & & & & - & $.44^{* *}$ \\
5. Desmotivación & 2.36 & 1.45 & & & & & - \\
\hline
\end{tabular}

Nota. ${ }^{* *} \mathrm{p}<.01 ;{ }^{*} \mathrm{p}<.05 ; M=$ Media; $D T=$ Desviación típica; $\mathrm{M} .=$ Motivación; $\mathrm{R} .=$ Regulación

\subsection{Comparación de la motivación en EF en función del género}

Al comparar las medias de la motivación en función del género de los participantes, se puede observar que las niñas se encuentras más motivadas intrínsecamente que los niños (se encontraron diferencias estadísticamente significativas). En el resto de tipos de motivación hay diferencias en las puntuaciones medias, aunque no fueron estadísticamente significativas $(p<.05)$.

Tabla 2. Diferencias entre las variables estudiadas en función del género

\begin{tabular}{c|c|c|c|c}
\hline Variable & Género & $\boldsymbol{M}$ & $\boldsymbol{D T}$ & $\mathbf{F}$ \\
\hline Motivación intrínseca & Niños $(\mathrm{n}=47)$ & 5.91 & 1.02 & $1.63^{\text {** }}$ \\
& Niñas $(\mathrm{n}=47)$ & 6.36 & .76 & \\
\hline Regulación Identificada & Niños $(\mathrm{n}=47)$ & 6.28 & .70 & 4.63 \\
& Niñas $(\mathrm{n}=47)$ & 6.40 & .56 & \\
\hline Regulación Introyectada & Niños $(\mathrm{n}=47)$ & 4.38 & 1.38 & \multirow{2}{*}{3.32} \\
& Niñas $(\mathrm{n}=47)$ & 3.85 & 1.58 & \\
\hline Regulación Externa & Niños $(\mathrm{n}=47)$ & 3.28 & 1.38 & .01 \\
\hline Desmotivación & Niñas $(\mathrm{n}=47)$ & 3.39 & 1.62 & \\
\hline & Niños $(\mathrm{n}=47)$ & 2.57 & 1.40 & 1.49 \\
\hline
\end{tabular}

Nota. ${ }^{\star \star} p<.01 ;{ }^{*} \mathrm{p}<.05 ; M=$ Media; $D T=$ Desviación típica

\subsection{Estadísticos descriptivos de la actividad físico-deportiva extraescolar}

Respecto del deporte o actividad física practicados durante el horario extraescolar, se puede ver que la actividad deportiva más practicada son el fútbol $(16 \%)$ y el baloncesto $(9,6 \%)$, seguidos de la natación. Pero nos encontramos que el $25.5 \%$ del alumnado no practican ningún tipo de deporte o actividad física extraescolar en su tiempo libre.

Tabla 3. Frecuencia de respuesta y porcentaje sobre si practican o no actividades físico-deportivas extraescolares

\begin{tabular}{lll}
\hline Respuesta & Frecuencia & Porcentaje \\
\hline No contesta & 4 & 4,3 \\
\hline Ninguno & 24 & 25,5 \\
\hline Fútbol & 15 & 16,0 \\
\hline Baloncesto & 9 & 9,6 \\
\hline
\end{tabular}




\begin{tabular}{lll}
\hline Natación & 7 & 7,4 \\
\hline Boxeo y fútbol & 1 & 1,1 \\
\hline Voleibol y futbol & 1 & 1,1 \\
\hline Natación y ciclismo & 2 & 2,1 \\
\hline Natación, ciclismo y atletismo & 1 & 1,1 \\
\hline Fútbol y pádel & 1 & 1,1 \\
\hline Atletismo & 3 & 3,2 \\
\hline Correr y baloncesto & 1 & 1,1 \\
\hline Fútbol y tenis & 1 & 1,1 \\
\hline Kárate y correr & 1 & 1,1 \\
\hline Multideportiva & 3 & 3,2 \\
\hline Patinaje y natación & 1 & 1,1 \\
\hline Senderismo & 1 & 1,1 \\
\hline Gimnasia rítmica & 5 & 5,3 \\
\hline Ciclismo y baile & 1 & 1,1 \\
\hline Pádel y baile & 1 & 1,1 \\
\hline Baile moderno & 5 & 5,3 \\
\hline Judo & 1 & 1,1 \\
\hline Voleibol & 1 & 1,1 \\
\hline Baloncesto y baile flamenco & 1 & 1,1 \\
\hline Natación y baile & 3 & 3,2 \\
\hline Total & $\mathbf{9 4}$ & $\mathbf{1 0 0}, 0$ \\
\hline
\end{tabular}

\section{DISCUSIÓN}

El objetivo principal de este trabajo fue analizar la motivación en las clases de Educación Física en Primaria. En este sentido, los resultados encontrados en general son positivos, puesto que las formas de motivación más autodeterminadas obtuvieron los valores medios más altos (motivación intrínseca y regulación identificada) y la desmotivación presentó valores relativamente bajos. Esto coincide con lo encontrado en otros estudios con alumnos de EF de Primaria (Cecchini et al., 2012; Cechini et al., 2013).

Los resultados de las correlaciones bivariadas son similares a los encontrados por Almagro et al. (2015) con alumnado de EF de Secundaria. Al comparar los resultados de la correlaciones con estudios con muestras similares, se puede observar similitudes y diferencias con el trabajo de Cecchini et al. (2013), ya que en ambos estudios la regulación introyectada y la regulación externa correlacionaba positivamente con la desmotivación. Sin embargo, en este trabajo no se encontraron correlaciones negativas y estadísticamente significativas entre la motivación intrínseca y la regulación identificada con la desmotivación como mostraron los resultados de Cecchini et al. (2013). Esto se puede deber a que la fiabilidad obtenida en el factor motivación intrínseca de esta investigación, ya que no se obtuvieron los niveles de fiabilidad deseados (alfa de Cronbach mayor a .07). En cualquier caso, estas relaciones deberán ser estudiadas con más profundidad en un futuro.

En cuanto a las diferencias obtenidas en función del género, llama la atención que en este estudio se encontraron diferencias estadísticamente significativas a favor de las alumnas en la motivación intrínseca. Sin embargo, en estudios como el de Amado et al. (2014) los resultados mostraron que los chicos presentan puntuaciones más altas en la motivación intrínseca que las chicas. Aunque estas diferencias pueden 
ser justificadas fácilmente, ya que aunque se midió la motivación deportiva en edad escolar no se evaluó la motivación en las clases de EF y como ya han mostrado algunos estudios las motivaciones son diferentes en función del contexto o ámbito de actividad física en el que nos encontremos (Moreno-Murcia et al., 2014).

Por otro lado, la mayoría de estudios que analizan la diferencias motivacionales en función del género se han realizado en alumnado de secundaria (e.g. Aspano, Lobato, Leyton, y Jiménez, 2015) o en adolescentes en el contexto deportivo (e.g. Moreno, Cervelló, y González-Cutre, 2007; Isorna, Rial, y Vaquero-Cristóbal, 2014). Por lo que sería interesante seguir profundizando en la motivación en las clases de EF de Primaria.

Un aspecto preocupante en nuestra sociedad actual es la falta de interés o el descenso del número de jóvenes que realizan algún deporte o actividad física, lo que ha hecho que muchos estudios se centren en analizar la cantidad personas que practican actividades deportivas (e.g. García-Ferrando, 2006; García-Ferrando y LLopis, 2011). En este sentido, en esta investigación se obtuvo que un $25.5 \%$ no realizaba ningún tipo de actividad física o deportiva fuera del horario escolar. Lógicamente, esto no se debe solamente a la motivación experimentada en las clases de EF en el colegio, sino que también puede deberse a otros factores que habría que analizar en futuros estudios con detenimiento: adquisición de hábitos más sedentarios (vídeojuegos, televisión, Internet, etc.), falta de oferta deportiva atractiva para los jóvenes, ausencia de apoyo parental, preferencia por otras actividades, etcétera. Sin embargo, lo que sí ha mostrado la investigación es que la motivación experimentada cuando practico EF puede influir en la intención de practicar deporte en el tiempo libre (Almagro et al., 2015; Méndez-Giménez et al., 2012).

En definitiva, este trabajo ha mostrado que la motivación en las clases de EF del alumnado encuestado es positiva (mayores puntuaciones en las formas de motivación más autodeterminadas). Además, en este centro las alumnas de tercer ciclo (5 y $6^{\circ}$ de Primaria) tienen una mayor motivación intrínseca (en la mayoría de los casos). Por último, se ha mostrado que un $25.5 \%$ del alumnado del centro estudiado no realizan actividades deportivas extraescolares y que los deportes más populares entre ellos son el fútbol, el baloncesto y la natación.

Por todo esto es necesario que los docentes estén formados en temáticas referentes a la motivación y que continúen con una formación continua adecuada, ya que esta falta de interés por realizar cualquier actividad física o deportiva, se puede combatir desde la escuela, ya que como afirman González-Cutre et al. (2011, p. 679):

Uno de los principales objetivos de las clases de Educación Física debe ser fomentar entre el alumnado la práctica de actividad física de forma regular. La Educación Física supone un medio excelente para desarrollar conocimientos y actitudes positivas hacia la práctica físico-deportiva.

Por último, este estudio presenta algunas limitaciones como son: un tamaño de muestra reducido, empleo de muestreo intencional o por conveniencia, uso de un cuestionario auto-administrado en un alumnado tan joven, etc. Dichas limitaciones, deberán ser tenidas en cuenta y tratar de ser subsanadas en futuras investigaciones. 


\section{CONCLUSIONES}

Los resultados de este estudio han mostrado que la motivación en las clases de EF del alumnado encuestado es positiva (mayores puntuaciones en las formas de motivación más autodeterminadas). Además, las alumnas de tercer ciclo (5ํy $6^{\circ}$ de Primaria) obtuvieron una mayor motivación intrínseca que los alumnos (las diferencias fueron estadísticamente significativas). No se encontraron diferencias estadísticamente significativas en función del género en el resto de regulaciones motivacionales. Por otro lado, se encontró que el $25.5 \%$ del alumnado participante en el estudio no realizaban actividades deportivas extraescolares; asimismo los deportes más practicados fueron: el fútbol, el baloncesto y la natación.

\section{REFERENCIAS}

Almagro, B. J., Navarro, I., Paramio, G., y Sáenz-López, P. (2015). Consecuencias de la motivación en las clases de educación física. Revista Digital de Educación Física EmásF, 34, 26-41. Recuperado de http://emasf2.webcindario.com/EmasF 34.pdf

Amado, D., Sánchez-Miguel, P. A., Leo, F. M., Sánchez-Oliva, D., y García-Calvo, T. (2014). Diferencias de género en la motivación y percepción de utilidad del deporte escolar. Revista Internacional de Medicina y Ciencias de la Actividad Física $y$ el Deporte, 14(56), 651-664. Recuperado de http://cdeporte.rediris.es/revista/revista56/artdiferencias510.htm

Aspano, M. I., Lobato, S., Leyton, M., y Jiménez, R. (2015). La motivación y los estilos de vida saludables en adolescentes pacenses en función del género. En $F$. Ruiz-Juan, J. Sánchez, M. Sánchez y J. Castro (Coords.), Libro de actas del XI Congreso Internacional sobre la enseñanza de la Educación Física y el deporte escolar (pp. 38-46). Almería: FEADEF-APEF.

Cecchini, J. A., Fernández-Losa, J. L., González, C., Cecchini, C. (2013). Aplicaciones del modelo de autodeterminación en la educación física de primaria. Revista Latinoamericana de Psicología, 45(1), 97-109.

Cecchini, J. A., Fernández-Losa, J. L., González, C., Fernández, J., y Méndez, A. (2012). La caída de la motivación autodeterminada en jóvenes escolares. SPORT TK: Revista Euroamericana de Ciencias del Deporte, 1(1), 25-31.

Deci, E. L., y Ryan, R. M. (1985). Intrinsic motivation and self-determination in human behavior. New York, USA: Plenum Press.

Deci, E. L., y Ryan, R. M. (2000). The "what" and "why" of goal pursuits: Human needs and the self-determination of behaviour. Psychological Inquiry, 11, 227-268.

Deci, E. L., y Ryan, R. M. (2008). Facilitating optimal motivation and psychological wellbeing across life's domains. Canadian Psychology, 49(1), 14-23.

Deci, E. L., y Ryan, R. M. (2012). Self-determination theory. En A. W. Kruglanski, P. A. M. Van Lange, y E. T. Higgins (Eds.), Handbook of theories social psychology (Vol. 1, pp. 416-437). London: SAGE. 
Deci, E. L., y Ryan, R. M. (2014). Autonomy and need satisfaction in close relationships: Relationships Motivation Theory. En N. Weinstein (Ed.), Human motivation and interpersonal relationships: Theory, research, and aplicattions (pp. 53-73). New York: Springer.

García-Ferrando, M. (2006). Veinticinco años de análisis del comportamiento deportivo de la población española (1980-2005). Revista Internacional de Sociología, 64 (44), 15-38.

García-Ferrando, y LLopis, R. (2011). Encuesta sobre los hábitos deportivos en España 2010. Ideal democrático y bienestar personal. Madrid: Consejo Superior de Deportes y Centro de Investigaciones Sociológicas. Recuperado de http://www.csd.gob.es/csd/estaticos/dep-soc/encuesta-habitosdeportivos2010.pdf

González-Cutre, D., Sicilia, Á., y Moreno, J. A. (2011). Un estudio cuasi-experimental de los efectos del clima motivador tarea en las clases de Educación Física. Revista de Educación, 356, 677-700.

Goudas, M., Biddle, S. J. H., y Fox, K. (1994). Perceived Locus of Causality, Goal Orientations and Perceived Competence in School Physical Education Classes. British Journal of Educational Psychology, 64, 453-463.

Gutiérrez, M., y López, E. (2012). Motivación, comportamiento de los alumnos y rendimiento académico. Infancia y Aprendizaje, 35, 61-72.

Hein, V., Müür, M., y Koka, A. (2004). Intention to be physically active after school graduation and its relationship to three types of intrinsic motivation. European Physical Education Review, 10, 5-19.

Isorna, M., Rial, A., y Vaquero-Cristóbal, R. (2014). Motivaciones para la práctica deportiva en escolares federados y no federados. Retos, 25, 80-84.

Lim, B. S. C., y Wang, C. K. J. (2009). Perceived autonomy support, behavioural regulations in physical education and physical activity intention. Psychology of Sport and Exercise, 10, 52-60.

Méndez-Giménez, A., Cecchini, J. A., Fernández-Río, J., y González, C. (2012). Autodeterminación y metas sociales: un modelo estructural para comprender la intención de práctica, el esfuerzo y el aburrimiento en educación física. Aula Abierta, 40, 51-62.

Méndez-Giménez, A., Fernández-Río, J., y Cecchini-Estrada, J. A. (2013). Climas motivacionales, necesidades, motivación y resultados en Educación Física. Aula Abierta, 41, 63-72.

Moreno, J. A., Cervelló, E., y González-Cutre, D. (2007). Analizando la motivación en el deporte: un estudio a través de la teoría de la autodeterminación. Apuntes de Psicología, 25, 35-51.

Moreno, J. A., González-Cutre, D., y Chillón, M. (2009). Preliminary validation in Spanish of a scale designed to measure motivation in physical education classes: The Perceived Locus of Causality (PLOC) Scale. The Spanish Journal of Psychology, 12, 327-337.

Moreno, J. A., González-Cutre, D., y Ruiz, L. M. (2009). Self-determination motivation and pshysical education importance. Human Movement, 10(1), 5-11. doi: 10.2478/v10038-008-0022-7

Moreno, J. A., Hernández, A., y González-Cutre, D. (2009). Complementando la teoría de la autodeterminación con las metas sociales: un estudio sobre la diversión en educación física. Revista Mexicana de Psicología, 26, 213-222. 
Ntoumanis, N., y Standage, M. (2009). Motivation in physical education classes: a selfdetermination theory perspective. Theory and Research in Education, 7, 194202.

Ryan, R. M., y Deci, E. L. (2000). Self-determination theory and the facilitation of intrinsic motivation, social development and well-being. American Psychologist, 55, 68-78.

Ryan, R. M., Williams, G. C., Patrick, H., y Deci, E. L. (2009). Self-determination theory and physical activity: The dynamics of motivation in development and wellness. Hellenic Journal of Psychology, 6, 107-124.

Vallerand, R. J., y Rousseau, F. L. (2001). Intrinsic and extrinsic motivation in sport and exercise: A review using the hierarchical model of intrinsic and extrinsic motivation. En R. N. Singer, H. A. Hausenblas, y C. M. Janelle (Eds.), Handbook of sport psychology (2 ${ }^{\mathrm{a}}$ ed., pp. 389-416). Nueva York: John Wiley \& Sons.

Vansteenkiste, M., Niemiec, C., y Soenens, B. (2010). The development of the five minitheories of self-determination theory: An historical overview, emerging trends, and future directions. En T. Urdan y S. Karabenick (Eds.), Advances in motivation and achievement, vol. 16: The decade ahead (pp.105-166). UK: Emerald Publishing. 